\title{
NÂNG CAO CHẤT LƯợNG ĐÀO TẠO NGUỒN NHÂN LỰC CỦA TRƯỜnG ĐẠI HỌC CÔNG NGHIẸP THÀNH PHỐ HỒ CHÍ MINH TRONG BỐI CẢNH HộI NHẬP
}

\author{
LÊ THỊ KIM HOA, HÀ TRỌNG QUANG \\ Khoa Quản trị kinh doanh, Trương Đại học Công nghiệp Thành phố Hồ Chi Minh \\ lethikimhoa@iuh.edu.vn
}

Tóm tắt - Nâng cao chất lượng đào tạo nguồn nhân lực của trường Đại học Công nghiệp Thành phố Hồ Chí Minh (IUH) trên cơ sở xuất phát từ những tiềm năng, thế mạnh trong đào tạo của nhà trường về đội ngũ giảng viên, tổ chức quản lý đào tạo, cơ sở vật chất phục vụ đào tạo, đánh giá kết quả học tập,... Trên cơ sở phân tích thực trạng chất lượng nguồn nhân lực của IUH trong thời gian qua, từ đó nhận diện các yếu tố ảnh hưởng tới chất lượng đào tạo nguồn nhân lực tại IUH, góp phần định hướng, xây dựng các giải pháp nâng cao chất lượng đào tạo nguồn nhân lực của IUHH trong bối cảnh hội nhập kinh tế quốc tế, khu vực và toàn cầu.

Từ khóa - Nâng cao; chất lượng; đào tạo; nguồn nhân lực; Đại học Công nghiệp TP HCM.

\section{IMPROVING THE QUALITY OF HUMAN RESOURCES TRAINING OF INDUSTRIAL UNIVERSITY HO CHI MINH CITY IN THE CONTEXT OF INTEGRATION}

\begin{abstract}
Improving the quality of human resources training of Industrial University Ho Chi Minh City (IUH) based on the potential and strength of the training of the university: training teachers, training management, facilities for training, evaluation of learning results,... On the basis of the analysis of the status of human resources quality of IUH in the past, identify the factors impacting the quality of human resource training at IUH, contribute to orientation and developing solutions to improve the quality of IUH human resources training in the context of international economic integration, regional and global integration.
\end{abstract}

Key words - Advanced; quality; training; human resources; Idustrial University Ho Chi Minh City.

\section{GIỚI THIẸU}

Chất lượng luôn là vấn đề quan trọng trong giáo dục đào tạo nói chung và trong các trường đại học nói riêng. Việc nâng cao chất lượng đào tạo là nhiệm vụ quan trọng nhất của bất kỳ cơ sở đào tạo đại học nào, là điểu kiện tiên quyết cho sự tồn tại và phát triển của các đơn vị đào tạo. Vì vậy, đề tài được hình thành để nghiên cứu sự hài lòng của sinh viển nhằm nâng cao chất lượng đào tạo nguồn nhân lực của trường Đại học Công nghiệp thành phố Hồ Chí Minh trong bối cảnh hội nhập cũng như mức độ ảnh hưởng của từng yếu tố. Từ đó, đưa ra một số kiến nghị cho Nhà trường, cán bộ quản lý và cán bộ giảng dạy nhằm nâng cao hơn nữa chất lượng đào tạo tại trường Đại học Công nghiệp thành phố Hồ Chí Minh.

Thách thức gay gắt nhất đối với giáo dục hiện nay là giữa yêu cầu phát triển quy mô với các điều kiện đảm bảo chất lượng. Thực tế cho thấy, do điều kiện kinh tế còn khó khăn, ngân sách hàng năm đầu tư cho giáo dục nói chung và giáo dục đại học nói riêng còn thấp; không đủ trang trải cho những yêu cầu tối cần thiết về các điều kiện đảm bảo như: trường sở, thư viện, phòng thí nghiệm, nhà xưởng thực hành,... Bên cạnh đó, yêu cầu nhanh chóng chuẩn hóa, hiện đại hóa, hội nhập giáo dục nước ta với các nước trong khu vực và trên thế giới cũng như việc đổi mới chương trình, nội dung, phương pháp giảng dạy đang đòi hỏi có những nỗ lực và quyết tâm cao.

Trong những năm gần đây, hệ thống giáo dục đại học nước ta không ngừng phát triển cả về qui mô và chất lượng. Trong hệ thống đại học công lập có: Đại học quốc gia, đại học vùng, trường đại học trọng điểm, đại học đa ngành, chuyên ngành, đại học mở, đại học cộng đồng. Trong mạng lưới đại học ngoài công lập có đại học bán công và dân lập. Nhiều trường đại học đang được hiện đại hóa từ cơ sở vật chất kĩ thuật đến đội ngũ cán bộ, giáo viên, nội dung chương trình đào tạo,... Tuy nhiên, nhìn nhận một cách khách quan, giáo dục đại học nước ta đang đứng trước nhiều khó khăn, thách thức, ảnh hưởng trực tiếp đến chất lượng đào tạo và việc nâng cao chất lượng đào tạo như: chất lượng đào tạo còn thấp, chưa đáp ứng được nhu cầu 
của xã hội, nhiều sinh viên ra trường chưa tìm được được việc làm đúng chuyên ngành,... trong đó trường ta không phải là trường hợp ngoại lệ.

Nhận diện những nhân tố ảnh hưởng tới chất lượng đào tạo nguồn nhân lực tại IUH, bài viết giúp làm rõ hơn cơ sở khoa học với kỳ vọng từng bước cải thiện và nâng cao chất lượng đào tạo nguồn nhân lực của nhà trường trong bối cảnh hội nhập kinh tế quốc tế, khu vực và toàn cầu.

\section{CƠ SỞ LÝ THUYẾT VÀ PHƯƠNG PHÁP NGHIÊN CÚU}

\subsection{Co' sở lý thuyết}

Khái niệm "chất lượng giáo dục đại học" hay "chất lượng trong giáo dục đại học" có nhiều cách hiểu khác nhau, nguyên nhân là do cách tiếp cận vấn đề của các nhà nghiên cứu. Tuy nhiên, theo định nghĩa của Green và Harvey (1993) chất lượng giáo dục đại học được đánh giá có tính khái quát và hệ thống. Green \& Harvey đã đề cập đến năm khía cạnh của chất lượng giáo dục đại học: chất lượng là sự vượt trội (hay sự xuất sắc); là sự hoàn hảo (kết quả hoàn thiện, không sai sót); là sự phù hợp với mục tiêu (đáp ứng nhu cầu của khách hàng); là sự đáng giá về đồng tiền (trên khía cạnh đánh giá để đầu tư); là sự chuyển đổi (sự chuyển đổi từ trạng thái này sang trạng thái khác). Trong số các định nghĩa trên, định nghĩa: "chất lượng là sự phù hợp với mục tiêu" đang được sử dụng bởi nhiều cơ quan đảm bảo chất lượng trên thế giới như: Hoa Kỳ, Anh Quốc và các nước Đông Nam Á,...

Có nhiều công trình nghiên cứu thực tiễn về mối quan hệ giữa chất lượng dịch vụ và sự thỏa mãn của khách hàng. Cronin \& Taylor (1992) đã kiểm định mối quan này và kết luận rằng: cảm nhận chất lượng dịch vụ dẫn đến sự thỏa mãn của khách hàng. Các nghiên cứu đã kết luận rằng: chất lượng dịch vụ là tiền đề của sự thỏa mãn và là nhân tố chủ yếu ảnh hưởng đến sự thỏa mãn. Do đó, muốn nâng cao sự hài lòng của khách hàng, nhà cung cấp dịch vụ phải nâng cao chất lượng dịch vụ. Nói cách khác, chất lượng dịch vụ và sự hài lòng của khách hàng có mối quan hệ tương hỗ chặt chẽ với nhau, trong đó chất lượng dịch vụ là cái được tạo ra trước và sau đó quyết định đến sự hài lòng của khách hàng. Mối quan hệ nhân quả giữa hai yếu tố này là vấn đề then chốt trong hầu hết các nghiên cứu về sự hài lòng của khách hàng.

Còn đối với khái niệm sự hài lòng, Bachelet (1995) cho rằng sự hài lòng của khách hàng như một phản ứng mang tính cảm xúc của khách hàng đáp lại với kinh nghiệm của họ với một sản phẩm hay một dịch vụ. Theo Zeithaml and Bitner (2000), sự hài lòng của khách hàng là sự đánh giá của khách hàng về một sản phẩm hay một dịch vụ đã đáp ứng được nhu cầu và mong đợi của họ. Còn theo Kotler (2001), sự hài lòng là mức độ của trạng thái của một người, bắt nguồn từ việc so sánh kết quả thu được từ sản phầm và những kỳ vọng của người đó. Theo Parasuraman, Zeithaml, Berry (1991), có 5 nhân tố ảnh hưởng đến sự hài lòng của khách hàng (1) Độ tin cậy (Reliability), (2) Mức độ đáp ứng (Responsiveness), (3) Sự đảm bảo (Assurance), (4) Sự cảm thông (Empathy), (5) Phương tiện hữu hình (Tangible).

Theo tác giả Brochado (2015), mô hình HEdPERF phân biệt các khía cạnh phi học thuật, khía cạnh học thuật, khía cạnh về chương trình đào tạo, truy cập, và uy tín trong dịch vụ giáo dục cao hơn, nó có mối tương quan cao với sự hài lòng tổng thể của sinh viên.

Theo một nghiên cứu về sự hài lòng của sinh viên về chất lượng đào tạo đại học là "Measuring student satisfaction with their studies in an International and European Studies Departerment" - của 2 tác giả G.V.Diamantis và V.K. Benos, trường đại học Piraeus - Hy Lạp thực hiện năm 2007. Nhóm tác giả này cho rằng sự hài lòng của sinh viên về khóa học là rất quan trọng và phụ thuộc vào nhiều yếu tố như: chương trình đào tạo, các môn học được giảng dạy, đội ngũ giảng viên, giáo trình, kinh nghiệm xã hội và cả kinh nghiệm trí tuệ. Để đánh giá sự hài lòng của sinh viên, nhóm tác giả này sử dụng phương pháp đánh giá sự hài lòng khách hàng MUSA (Multicriteria Satisfaction Analysis - phân tích sự hài lòng nhiều tiên chí) gồm 5 tiêu chí: chương trình đào tạo, giảng dạy, hữu hình, hỗ trợ hành chính, hình ảnh của trường.

Theo nghiên cứu của các tác giả Nguyễn Thúy Quỳnh Loan và Nguyễn Thị Thanh Thoản về đề tài "Đánh giá chất lượng đào tạo dưới góc độ sinh viên trường ĐH Bách Khoa TPHCM". Đề tài nghiên cứu các nhân tố liên quan như: chương trình đào tạo, đội ngũ giảng viên, cơ sở vật chất, kết quả đào tạo. Đề tài nghiên cứu cho thấy sinh viên đánh giá cao về chương trình, giảng viên, cơ sở vật chất nhưng về yêu cầu liên hệ thực tế thì chưa được hài lòng cao.

\subsection{Phương pháp nghiên cứu}

Nghiên cứu của đề tài sẽ được thực hiện thông qua 2 bước: (1) nghiên cứu sơ bộ, tác giả dùng phương pháp định tính và (2) nghiên cứu chính thức, tác giả dùng phương pháp nghiên cứu định lượng. Nghiên cứu định tính được thiết kế một cách có tính chất thăm dò tự nhiên vấn đề nghiên cứu, khám phá trực tiếp các ý tưởng 
và dùng để mô tả trong phạm vi bảng câu hỏi sơ bộ, cố gắng giải thích sự tương quan có ý nghĩa từ các thang đo trong mô hình nghiên cứu. Từ kết quả này, tác giả xây dựng bảng câu hỏi chính thức được hình thành sao cho phù hợp về mặt ý nghĩa các thang đo và các đối tượng chọn lấy mẫu. Nghiên cứu định lượng được thiết kế với dữ liệu được thu thập thông qua bảng câu hỏi nghiên cứu chính thức.

Phương pháp thu thập số liệu: Số liệu của nghiên cứu được thu thập bằng cách phỏng vấn trực tiếp các sinh viên năm cuối trường Đại học Công nghiệp TP Hồ Chí Minh theo phương pháp phân tầng kết hợp với ngẫu nhiên. Hiện nay, theo nhiều nhà nghiên cứu, kích thước mẫu càng lớn càng tốt (Nguyễn, 2011). Hair et al. (2006) cho rằng để sử dụng phân tích nhân tố khám phá (EFA), kích thước mẫu tối thiểu phải là 50 , tốt hơn là 100 và tỉ lệ quan sát/biến đo lường là $5: 1$, nghĩa là 1 biến đo lường cần tối thiểu 5 quan sát (Nguyễn, 2011). Cụ thể, trong mô hình nghiên cứu được nhóm nghiên cứu đề xuất có 24 biến quan sát có thể được sử dụng trong phân tích nhân tố khám phá. Do đó, số mẫu tối thiểu cần thiết của nghiên cứu là $24 \times 5=120$ mẫu. Thực tế, nhóm tác giả đã tiến hành điều tra 294 sinh viên trong khoảng thời gian từ tháng 09/2018 đến tháng 10/2018. Như vậy, số liệu được thu thập đảm bảo thực hiện tốt mô hình nghiên cứu.

\section{Nhân tố 1: Chưong trình đào tạo (CTÐT) kí hiệu là X1 bao gồm 9 biến quan sát}

- Khối lượng kiến thức chương trình đào tạo phù hợp (CTĐT1)

- Nội dung chương trình đào tạo phù hợp với mục tiêu đào tạo của ngành (CTĐT2)

- Nội dung giảng dạy mang tính thực tiễn cao (СТĐT3)

- Chương trình đào tạo đáp ứng nhu cầu nhân lực của xã hội (CTĐT4)

- Tỷ lệ phân bổ giữa lý thuyết và thực hành phù hợp với ngành học (CTĐT5)

- Chương trình đào tạo cho phép sinh viên linh hoạt sắp xếp thời gian học (CTĐT6)

- Chương trình đào tạo được thiết kế có thể liên thông với các trình độ đào tạo và chương trình giáo dục khác (CTÐТ7)

- Giáo trình mỗi môn được cung cấp đầy đủ, đa dạng và thống nhất (CTĐT8)

- Đào tạo các kỹ năng quan trọng như kỹ năng giao tiếp, kỹ năng đàm phán, kỹ năng xử lý tình huống (СТĐТ9)

\section{Nhân tố 2: Đội ngũ giảng viên (ĐNGV) kí hiệu là X2 và bao gồm 4 biến quan sát}

- Giảng viên sẵn sàng chia sẻ kinh nghiệm và kiến thức với sinh viên (ĐNGV1)

- Giảng viên hướng dẫn có sự quan tâm chân thành trong việc giải quyết vấn đề của sinh viên (ĐNGV2)

- Giảng viên có kiển thức sâu rộng về chuyên môn (ĐNGV3)

- Giảng viên đã sử dụng nhiều phương pháp giảng dạy (ĐNGV4)

Nhân tố 3: Hoạt động thục tế (HĐTT) kí hiệu là X3 và bao gồm 8 biến quan sát

- Tổ chức cho sinh viên tìm hiểu những nét kinh doanh đặc trưng của từng khu vực kinh tế (HĐTT1)

- Tổ chức thực tế ngoài trường để sinh viên tìm hiểu thêm về các loại hình doanh nghiệp (HĐTT2)

- Kết hợp thực tế ngoài trường với các bài tập thực hành (HĐTT3)

- Tổ chức các buổi tập huấn, thực hành các nghiệp vụ cho sinh viên (HĐTT4)

- Tổ chức các hoạt động ngoại khóa, đội nhóm rèn luyện kỹ năng (HĐTT5)

- Sinh viên được giảng dạy và tập huấn các nghiệp vụ cơ bản và cần thiết (HĐTT6)

- Nhà trường liên hệ với các cơ sở kinh doanh, tổ chức tạo điều kiện cho sinh viên thực tập, kiến tập (HĐTT7)

- Tạo điều kiện cho sinh viên kiến tập, thực hành nghiệp vụ, thực tập thường xuyên (HĐTT8)

Nhân tố 4: Cơ sở vật chất (CSVC) kí hiệu là X4 và bao gồm 3 biến quan sát

- Quy hoạch tổng thể của nhà trường thuận tiện cho hoạt động dạy và học (CSVC1)

- Nguồn tài liệu trong thư viện đáp ứng được nhu cầu của sinh viên (CSVC2)

- Thiết bị công nghệ phục vụ giảng dạy \& học tập hoạt động có hiệu quả (CSVC3)

Nhân tố 5: Múc độ hài lòng kí hiệu là Y và bao gồm 3 biến quan sát

- Chương trình đào tạo (Y1)

- Giảng viên (Y2)

- Cơ sở vật chất (Y3) 


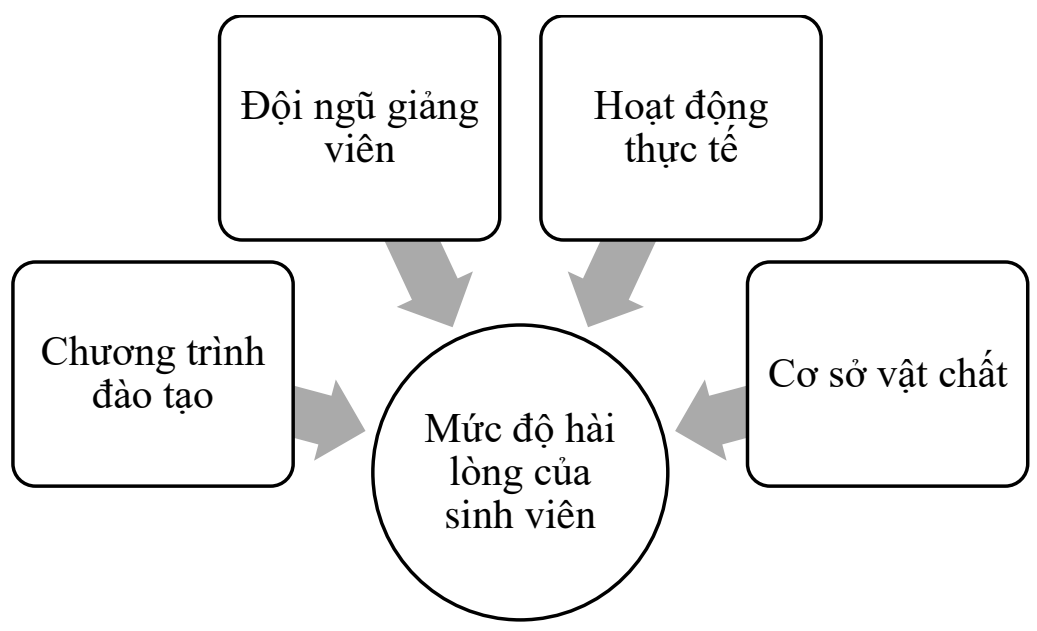

Hình 1: Mô hình nghiên cứu đề xuất

\section{KẾT QUẢ NGHIÊN CÚ̉U VÀ THẢO LUÂN}

Đề tài nghiên cứu được thực hiện thông qua hình thức phỏng vấn sinh viên trực tiếp. Phương pháp lấy mẫu thuận tiện với kích cỡ mẫu nghiên cứu là 250 sinh viên. Tác giả đã gửi đi 270 bảng câu hỏi khảo sát, thu về 250 bảng câu hỏi trả lời và thỏa mãn yêu cầu xử lý, kết quả thực hiện mô hình nghiên cứu như sau:

Các nhân tố trong mô hình nghiên cứu sau khi thực hiện kiểm định độ tin cậy của thang đo Cronbach's Alpha có 3 biến quan sát bị loại ra khỏi mô hình nghiên cứu vì không thỏa mãn điều kiện hệ số tương quan biến - tổng $>=0.3$ (Nunnally, 1978; Peterson, 1994; Slater, 1995) đó là các biến quan sát CTĐT6: Chương trình đào tạo cho phép sinh viên linh hoạt sắp xếp thời gian học; CTĐT7: Chương trình đào tạo được thiết kế có thể liên thông với các trình độ đào tạo và chương trình giáo dục khác; HĐTT4: Tổ chức các buổi tập huấn, thực hành các nghiệp vụ cho sinh viên. Các biến quan sát còn lại trong mô hình đạt yêu cầu khi phân tích độ tin cậy của thang đo nên đủ cơ sở để thực hiện các bước phân tích tiếp theo.

Bảng 1: Kết quả đánh giá độ tin cậy của thang đo

\begin{tabular}{ccccc}
\hline Nhân tố & $\begin{array}{c}\text { Trung bình thang đo } \\
\text { nếu loại biến }\end{array}$ & $\begin{array}{c}\text { Phương sai thang đo nếu Tương quan biến }- \\
\text { loại biến }\end{array}$ & $\begin{array}{c}\text { Hệ số Cronbach's } \\
\text { tổng }\end{array}$ & \begin{tabular}{c} 
Alpha nếu loại biến \\
\hline ĐNGV1
\end{tabular} $7^{2.69}$ \\
CTĐT1 & 72.78 & 124.430 & 0.625 & 0.929 \\
CTĐT2 & 72.64 & 125.191 & 0.458 & 0.933 \\
CTĐT3 & 72.76 & 123.528 & 0.644 & 0.928 \\
CTĐT4 & 72.68 & 125.226 & 0.607 & 0.929 \\
HĐTT1 & 72.73 & 121.212 & 0.708 & 0.927 \\
CTĐT5 & 72.80 & 123.732 & 0.629 & 0.929 \\
HĐTT2 & 72.56 & 122.966 & 0.619 & 0.929 \\
HĐTT3 & 72.52 & 122.902 & 0.622 & 0.929 \\
HĐTT4 & 72.48 & 124.415 & 0.579 & 0.930 \\
CSVC3 & 72.65 & 124.226 & 0.630 & 0.929 \\
ĐNGV2 & 72.48 & 123.525 & 0.603 & 0.929 \\
ĐNGV3 & 72.63 & 124.150 & 0.610 & 0.929 \\
ĐNGV4 & 72.59 & 125.027 & 0.611 & 0.929 \\
CTĐT8 & 72.62 & 124.758 & 0.620 & 0.929 \\
CTĐT6 & 72.57 & 125.470 & 0.601 & 0.929 \\
CTĐT7 & 72.81 & 124.385 & 0.730 & 0.927 \\
HĐTT6 & 72.68 & 125.730 & 0.553 & 0.930 \\
HĐTT7 & 72.71 & 124.686 & 0.625 & 0.929 \\
CTĐT9 & 72.74 & 125.929 & 0.562 & 0.930 \\
HĐTT8 & 72.64 & 126.569 & 0.517 & 0.931 \\
\hline
\end{tabular}

Nguồn: Kết quả kiểm định Cronbachs's Alpha tùu số liệu điều tra 
Kết quả phân tích nhân tố khám phá (EFA) sau 3 vòng với các kiểm định được đảm bảo như sau: (1) Độ tin cậy của các biến quan sát (Factor loading $>0,5) ;(2)$ Kiểm định tính phù hợp của mô hình $(0,5<\mathrm{KMO}$ $=0,828<1)$; (3) Kiểm định Barlett về tương quan của các biến quan sát (Sig. $<0,05)$; kiểm định phương sai cộng dồn $=67,15 \%$ (Cumulatine variance $>50 \%)$.

Bảng 2: Phân tích nhân tố các biến độc lập trong mô hình nghiên cứu

\begin{tabular}{|c|c|c|c|c|}
\hline \multirow{3}{*}{ Biến quan sát } & \multicolumn{4}{|c|}{ Ma trận nhân tố } \\
\hline & \multicolumn{4}{|c|}{ Nhân tố } \\
\hline & 1 & 2 & 3 & 4 \\
\hline HĐTT1 & .817 & & & \\
\hline HDTT3 & .771 & & & \\
\hline HĐTT6 & .753 & & & \\
\hline HĐTT7 & .723 & & & \\
\hline HĐTT2 & .669 & & & \\
\hline HĐTT8 & .654 & & & \\
\hline HĐTT5 & .605 & & & \\
\hline СТĐТ5 & & .850 & & \\
\hline СТĐТ1 & & .846 & & \\
\hline СТÐТ9 & & .796 & & \\
\hline СТÐТ2 & & .727 & & \\
\hline СТÐТ3 & & .616 & & \\
\hline СТĐТ4 & & .615 & & \\
\hline ĐNGV1 & & & .818 & \\
\hline ĐNGV3 & & & .772 & \\
\hline ĐNGV2 & & & .755 & \\
\hline ĐNGV4 & & & .735 & \\
\hline CSVC1 & & & & .840 \\
\hline $\mathrm{CSVC} 2$ & & & & .755 \\
\hline СТÐТ8 & & & & .728 \\
\hline CSVC3 & & & & .691 \\
\hline \multicolumn{5}{|c|}{ Meyer-Olkin Measure of San } \\
\hline
\end{tabular}

Kết quả phân tích nhân tố khám phá EFA cho thấy có 4 nhân tố được tạo thành có tác động đến mức độ hài lòng của sinh viên. Nhân tố $\mathrm{F} 1$ bao gồm các biến quan sát: Tổ chức cho sinh viên tìm hiểu những nét kinh doanh đặc trưng của từng khu vực kinh tế (HĐTT1); Tổ chức thực tế ngoài trường để sinh viên tìm hiểu thêm về các loại hình doanh nghiệp (HĐTT2); Kết hợp thực tế ngoài trường với các bài tập thực hành (HĐTT3); Tổ chức các hoạt động ngoại khóa, đội nhóm rèn luyện kỹ năng (HĐTT5); Sinh viên được giảng dạy và tập huấn các nghiệp vụ cơ bản và cần thiết (HĐTT6); Nhà trường liên hệ với các cơ sở kinh doanh, tổ chức tạo điều kiện cho sinh viên thực tập, kiến tập (HĐTT7); Tạo điều kiện cho sinh viên kiến tập, thực hành nghiệp vụ, thực tập thường xuyên (HĐTT8). Nhân tố F2 bao gồm các biến quan sát: Khối lượng kiến thức chương trình đào tạo phù hợp (CTĐT1); Nội dung chương trình đào tạo phù hợp với mục tiêu đào tạo của ngành (CTĐT2); Nội dung giảng dạy mang tính thực tiễn cao (CTĐT3); Chương trình đào tạo đáp ứng nhu cầu nhân lực của xã hội (CTĐT4); Tỷ lệ phân bổ giữa lý thuyết và thực hành phù hợp với ngành học (CTĐT5); Đào tạo các kỹ năng quan trọng như kỹ năng giao tiếp, kỹ năng đàm phán, kỹ năng xử lý tình huống (CTĐT9). Nhân tố $\mathrm{F} 3$ bao gồm các biến quan sát: Giảng viên sẵn sàng chia sẻ kinh nghiệm và kiến thức với sinh viên (ĐNGV1); Giảng viên hướng dẫn có sự quan tâm chân thành trong việc giải quyết vấn đề của sinh viên (ĐNGV2); Giảng viên có kiến thức sâu rộng về chuyên môn (ĐNGV3); Giảng viên đã sử dụng nhiều phương pháp giảng dạy (ĐNGV4). Nhân tố F4 bao gồm các biến qua sát: Quy hoạch tổng thể của nhà trường thuận tiện cho hoạt động dạy và học $(\mathrm{CSVC} 1)$; Nguồn tài liệu trong thư viện đáp ứng được nhu cầu của sinh viên (CSVC2); Thiết bị công nghệ phục vụ giảng dạy \& học tập hoạt động có hiệu quả (CSVC3); Giáo trình mỗi môn được cung cấp đầy đủ, đa dạng và thống nhất (CTĐT8). Mồ hình được hiệu chỉnh như sau: 


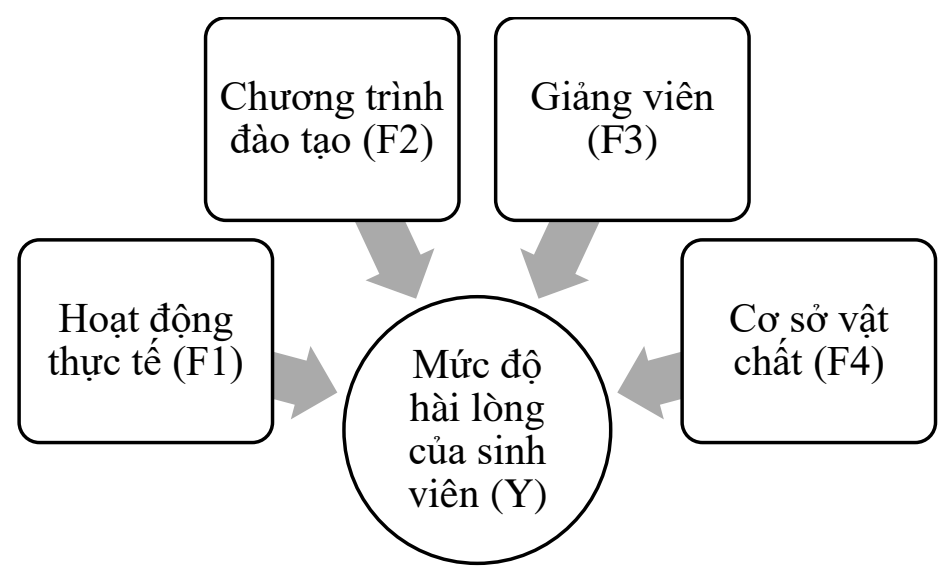

Hình 2: Mô hình nghiên cứu hiệu chỉnh

Bảng 3: Kết quả phân tích hồi quy tuyến tính

\begin{tabular}{|c|c|c|c|c|}
\hline Tên biến & Hệ số B & Hệ số Beta & Sig. & VIF \\
\hline Hằng số & 0,295 & - & 0,004 & - \\
\hline F1: Hoạt động thực tế & 0,187 & 0,186 & 0,001 & 1,991 \\
\hline F2: Chương trình đào tạo & 0,117 & 0,123 & 0,012 & 1,552 \\
\hline F3: Giảng viên & 0,358 & 0,382 & 0,000 & 1,032 \\
\hline F4: Cơ sở vật chất & 0,259 & 0,259 & 0,000 & 1,987 \\
\hline Hệ số Sig.F của mô hình & & 0,000 & & \\
\hline Hệ số R2 hiệu chỉnh & & 0,472 & & \\
\hline Hệ số Durbin - Watson & & 1,826 & & \\
\hline
\end{tabular}

Nguồn: Kết quả phân tích hồi quy tù số liệu điều tra Kết quả phân tích các hệ số hồi quy trong mô hình cho thấy, mức ý nghĩa của các nhân tố có Sig. (nhỏ hơn $0,05)$. Do đó, ta có thể nói rằng các biến độc lập đều có tác động đến sự hài lòng của sinh viên về chất lượng đào tạo. Giá trị R điều chỉnh (Adjusted $\mathrm{R}$ Square) phản ánh chính xác hơn sự phù hợp của mô hình đối với tổng thể, ta có giá trị $\mathrm{R}$ điều chỉnh bằng 0,472 (hay $47,2 \%$ ) có nghĩa tồn tại mô hình hồi qui tuyến tính giữa Sự hài lòng và 4 nhân tố trong chất lượng đào tạo. Phân tích phương sai ANOVA cho thấy trị số $\mathrm{F}$ có mức ý nghĩa Sig. $=0,000$ nhỏ hơn 0,05 có nghĩa là mô hình hồi qui phù hợp với dữ liệu thu thập được và các biến đưa vào đều có ý nghĩa trong thống kê với mức ý nghĩa $5 \%$. Kiểm định hiện tượng tự tương giữa các phần dư bằng hệ số Durbin-Watson, không có tương quan giữa các phần dư thì $1<$ Durbin-Watson $<3$, theo Hoàng Trọng và Chu Nguyễn Mộng Ngọc (2008). Kết quả phân tích có đại lượng thống kê Durbin-Watson $=1.873(1<1.826<3)$ cho thấy không có sự tương quan giữa các phần dư. Điều này có ý nghĩa là mô hình hồi quy không vi phạm giả định về tính độc lập của sai số. Phương trình hồi qui ước lượng các nhân tố ảnh hưởng đến mức độ hài lòng của sinh viên đối với chất lượng đào tạo được thiết lập như sau:

$\begin{array}{llll}\text { Sự hài lòng của sinh viên } \quad= & + & 0,186 & * \text { Hoạt động thực tế } \\ & + & 0,123 * \text { Chương trình đào tạo } \\ & + & 0,382 & * \text { Giảng viên } \\ & + & 0,259 * \text { Cơ sớ vật chất }\end{array}$

Dựa vào phương trình hồi qui cho thấy: bốn biến đưa vào mô hình đều có tương quan thuận với mức độ hài lòng của sinh viên đối với chất lượng đào tạo. Trong đó, nhân tố tác động mạnh nhất đển chất lượng đào tạo của sinh viên là giảng viên vì ảnh hưởng $38,2 \%$ đến sự hài lòng, tiếp đến là nhân tố cơ sở vật chất ảnh hưởng $25,9 \%$ đến sự hài lòng, nhân tố hoạt động thực tế ảnh hưởng $18,6 \%$ đến sự hài lòng và thấp nhất là nhân tố chương trình đào tạo ảnh hưởng $12,3 \%$ đến sự hài lòng. Mô hình cho thấy các biến độc lập đều ảnh hưởng thuận chiều đến mức độ hài lòng của sinh viên ở độ tin cậy $95 \%$. Qua phương trình hồi qui chưa chuẩn hóa chúng ta thấy, nếu giữ nguyên các biến độc lập còn lại không đổi thì khi điểm đánh giá về 
“chương trình đào tạo" tăng lên 1 điểm thì sự hài lòng của sinh viên tăng lên trung bình 0,117 điểm. Tương tự, khi điểm đánh giá về "đội ngũ giảng viên" tăng lên 1 điểm thì sự hài lòng của sinh viên về chất lượng đào tạo tăng lên trung bình 0,358 điểm; khi điểm đánh giá về hoạt động thực tế tăng lên 1 điểm thì sự hài lòng của sinh viên về chất lượng đào tạo tăng lên trung bình 0,187 điểm và cuối cùng khi điểm đánh giá về cơ sở vật chất tăng lên 1 điểm thì sự hài lòng của sinh viên về chất lượng đào tạo tăng lên trung bình 0,259 điểm.

Tóm lại, kết quả nghiên cứu thực nghiệm đã xác định 4 nhân tố tác động đến mức độ hài lòng của sinh viên đối với chất lượng đào tạo theo thứ tự mức độ quan trọng là: Mức độ tương tác của giảng viên, cơ sở vật chất của nhà trường, hoạt động thực tế của sinh viên và cuối cùng là chương trình đào tạo. Kết quả nghiên cứu đã cung cấp một cẳn cứ khoa học thực tiễn quí báu cho việc cải tiến chất lượng đào tạo của trường. Mức độ hài lòng của sinh viên càng cao, càng tạo sự hấp dẫn, lôi cuốn sinh viên say mê học tập, từ đó chất lượng đào tạo sẽ được nâng cao, đáp ứng thực tiễn nhu cầu xã hội.

\section{KẾT LUẬN VÀ HÀM Ý QUẢN TR!}

Mục đích chính của nghiên cứu là xác định các thành phần tác động vào sự hài lòng của sinh viên về chất lượng đào tạo của trường Đại học Công nghiệp Thành phố Hồ Chí Minh, xây dựng và đánh giá các thang đo lường các thành phần. Để khẳng định sự tác động của các thành phần này vào sự hài lòng của sinh viên về chất lượng đào tạo, một mô hình lý thuyết được xây dựng và kiểm định. Mô hình lý thuyết được xây dựng dựa trên cơ sở lý thuyết về chất lượng dịch vụ, chất lượng đào tạo, sự hài lòng của sinh viên và các thành phần tác động vào sự hài lòng.

Yếu tố giảng viên: Nâng cao chất lượng đội ngũ giảng viên bằng cách tạo điều kiện thuận lợi để giảng viên có cơ hội học tập, nghiên cứu chuyên môn trong và ngoài nước. Khuyến khích và hỗ trợ giảng viên tham dự các Hội thảo khoa học chuyên ngành với tư cách là người trình bày hoặc người tham gia để giảng viên được tiếp xúc, trao đổi các kiến thức mới. Bồi dưỡng giảng viên về ngoại ngữ, tin học, kỹ năng thiết kế bài giảng và kỹ năng sử dụng các phương tiện hiện đại, kỹ năng tìm kiếm và cập nhật thông tin trên Internet,... để cập nhật thông tin hữu ích cho việc giảng dạy đáp ứng nhu cầu phát triển kinh tế xã hội và nhu cầu phát triển nguồn nhân lực ngày càng cao của đất nước.

Đổi mới phương pháp giảng dạy theo phương châm lấy người học làm trung tâm, học đi đôi với hành, chuyển đổi mạnh mẽ từ dạy kiến thức là chủ yếu sang phương pháp khai thác thông tin, phương pháp nghiên cứu, ứng dụng là chủ yếu. Giảng viên tạo điều kiện tốt nhất cho sinh viên tiếp thu kiến thức mới một cách thuận lợi và hình thành, phát triển được khả năng tự học, tự nghiên cứu là vấn đề quan trọng nhất đối với giảng viên hiện nay. Thay vì sử dụng phương pháp thuyết trình nhàm chán để truyền đạt kiến thức cho sinh viên thì giảng viên có thể sử dụng phương pháp đàm thoại để hướng dẫn, gợi mở và dẫn dắt sinh viên đến với các kiến thức mới. Trong trường hợp này giảng viên không phải là người truyền thụ mà là người hướng dẫn để sinh viên tự tìm hiểu, tự nghiên cứu để lý giải được các vấn đề. Khi đó kiến thức sẽ tự động được hình thành trong sinh viên một cách tự nhiên, không gượng ép, không nhồi nhét và giúp sinh viên nhớ lâu hơn. Bên cạnh đó, giảng viên cũng nên kết hợp phương pháp làm việc nhóm với việc cho sinh viên tự thực hiện, tự trình bày các seminar, các chuyên đề khoa học để tăng cường khả năng tự học, tự nghiên cứu. Bằng phương pháp này các kiến thức kỹ năng cần thiết cho sinh viên sẽ được hình thành và phát triển toàn diện. Yếu tố cơ sở vật chất: Tăng cường công tác vệ sinh tại các khu vực nhà vệ sinh của các giảng đường, phòng học, khu sinh hoạt văn hóa, văn nghệ, thể dục thể thao. Tăng cường công tác an ninh, an toàn các khu vực của Nhà trường vào buổi tối. Thường xuyên kiểm tra, kịp thời sửa chữa hệ thống đèn chiếu sáng của hệ thống các phòng học, giảng đường. Bồ sung đèn chiếu sáng tại các khu vực thư viện, khu sinh hoạt văn hóa, trong khuôn viên trường. Đổi mới, nâng cấp hệ thống mạng Internet, máy chiếu, máy vi tính của hệ thống các giảng đường, phòng học và các phòng thực hành máy tính. Lắp đặt mạng Internet cho khu vực ký túc xá sinh viên. Tăng cường máy móc trang thiết bị hiện đại cho các phòng thí nghiệm chuyên ngành. Định kì kiểm tra khu vực căn tin, chế biến thức ăn nhằm đảm bảo công tác vệ sinh an toàn thực phẩm, giữ gìn sức khỏe cho sinh viên. Bổ sung các điều kiện phục vụ hoạt động văn hóa, văn nghệ, thể dục thể thao tại khu vực ký túc xá sinh viên. Xây dựng thêm nhà giữ xe phía cổng sau của Nhà trường. Nâng cấp nhà Thư viện, đầu tư thêm máy móc và trang thiết bị. Thư viện cần tăng cường cập nhật sách, báo, tài liệu phục vụ nhu cầu học tập, nghiên cứu khoa học của sinh viên. Tăng thời gian hoạt động của thư viện nhằm đáp ứng nhu cầu học tập, nghiên cứu của sinh viên. Nâng cấp hệ thống mạng wifi, đường truyền nhằm tạo điều kiện cho sinh viên có thể tra cứu tài liệu trong thời gian tự học tại trường. Mặt khác, nâng cấp đường truyền 
để giảm bớt những khó khăn, bất cập khi sinh viên đăng kí môn học. Đầu tư mua sắm thêm hệ thống máy vi tính ở các phòng ban, nâng cấp hệ thống thông tin, bảo mật thông tin, website, thư viện điện tử. Tiếp tục điều chỉnh, cập nhật thang đo sự hài lòng của sinh viên về đánh giá chất lượng đào tạo và định kỳ tổ chức khảo sát ý kiến sinh viên sau từng học kỳ nhằm có những giải pháp hiệu quả nhất cho quá trình cải tiến dịch vụ sinh viên, góp phần thực hiện tốt công tác đảm bảo chất lượng bên trong của nhà trường.

Yếu tố hoạt động thực tế: Với những lợi thế vốn có của trường là qui mô lớn và được nhiều người biết đến và đa phần sinh viên hài lòng cao về chất lượng đào tạo của trường. Do đó, hoạt động đào tạo cung cấp kiến thức, kỹ năng cho sinh viên đáp ứng nhu cầu sinh viên và tạo sự hấp dẫn đối với các Doanh nghiệp là một việc làm hết sức cần thiết. Chính vì vậy, Nhà trường cần "tạo dựng giá trị" nâng cao giá trị bằng cấp của trường Đại học Công nghiệp Thành Phố Hồ Chí Minh bằng cách nâng cao thương hiệu, hình ảnh, uy tín và chất lượng đào tạo của trường. Để đảm bảo danh tiếng chất lượng đào tạo, Nhà trường cần đo lường thành công của sinh viển đầu ra, những người đang xin việc hoặc đang làm tại các doanh nghiệp sau khi tốt nghiệp. Việc làm này cần được thực hiện và cải tiến liên tục dựa trên nhu cầu sinh viên và nhu cầu nhân lực hiện tại của xã hội. Chính vì thế, Nhà trường cần tổ chức cho sinh viên tìm hiểu những nét kinh doanh đặc trưng của từng khu vực kinh tế, tổ chức thực tế ngoài trường để sinh viên tìm hiểu thêm về các loại hình doanh nghiệp, kết hợp thực tế ngoài trường với các bài tập thực hành, tổ chức các buổi tập huấn, thực hành các nghiệp vụ cho sinh viên, tổ chức các hoạt động ngoại khóa, đội nhóm rèn luyện kỹ năng, sinh viên được giảng dạy và tập huấn các nghiệp vụ cơ bản và cần thiết, Nhà trường liên hệ với các cơ sở kinh doanh, tổ chức tạo điều kiện cho sinh viên thực tập, kiến tập và tạo điều kiện cho sinh viên kiến tập, thực hành nghiệp vụ, thực tập thường xuyên.

Ngoài ra, trường nên mời các doanh nghiệp, các nhà hoạt động thực tiễn tham gia giảng dạy nhằm trang bị cho sinh viên những kỹ năng làm việc khi tốt nghiệp, tổ chức những buổi tọa đàm giữa sinh viên và doanh nghiệp nhằm chia sẻ những kinh nghiệm trong làm việc, cũng như những kinh nghiệm trong quá trình tuyển dụng và trả lời phỏng vấn,...

Yếu tố về chương trình đào tạo: Xây dựng chương trình phù hợp với nhu cầu thực tế, nâng cao khả năng ứng dụng trong bối cảnh bùng nổ thông tin, công nghiệp hóa, hiện đại hóa và hội nhập quốc tế. Nhà trường cần giảm thời gian giảng dạy lý thuyết, tăng thời lượng nghiên cứu và thực hành, ứng dụng trong phân bổ thời gian giữa các môn học lý thuyết và các môn ứng dụng của mỗi ngành đào tạo, phân bổ thời lượng giữa hướng dẫn lý thuyết và thực hành ứng dụng cho từng môn học.

Tiếp tục duy trì và nâng cao trong việc thiết kế chương trình đào tạo cho phép sinh viên linh hoạt sắp xếp thời gian học tập theo cơ chế tín chỉ. Chương trình đào tạo được thiết kế theo hướng có thể liên thông với các trình độ đào tạo và chương trình giáo dục khác.

Giáo trình, tài liệu tham khảo phải đảm bảo đầy đủ về số lượng và chất lượng. Duy trì và nâng cao sự đầy đủ, đa dạng và thống nhất giáo trình mỗi môn. Nội dung giáo trình, tài liệu phải phù hợp với mục tiêu, chương trình đào tạo, đảm bảo chuẩn kiến thức, kỹ năng và chuẩn đầu ra. Kiến thức trong giáo trình, tài liệu phải được trình bày khoa học, logic, đảm bảo cân đối giữa lý luận và thực hành, phù hợp với thực tiễn và luôn cập nhật những tri thức mới nhất của nhân loại.

Nhà trường cần xây dựng các mối liên hệ chặt chẽ với các doanh nghiệp bên ngoài, tạo điều kiện cho sinh viên được tham quan, thực tập, tiếp xúc với môi trường thực tế. Bên cạnh đó mối liên hệ này cũng giúp Nhà trường nắm được các nhu cầu sử dụng lao động mà thiết kế chương trình học sát với yêu cầu thực tế của các doanh nghiệp. Có như thế thì chương trình đào tạo mới thường xuyên được đổi mới, phù hợp với nhu cầu nhân lực của xã hội và đáp ứng được nhu cầu học tập, nghiên cứu của sinh viên.

\section{TÀI LIỆU THAM KHẢO}

[1] Allen \& Rao (2000), “Analysis of Customer Satisfaction Data”, ASQ Quality Press, Milwaukee.

[2] Bùi Thị Hằng, (2016), "Nâng cao chất lượng đội ngũ giảng viên trường Đại học Tài chính - Quản trị kinh doanh”, Luận văn thạc sĩ.

[3] Mai Văn Nam (2008), "Kinh tế lượng (Econometrics)", Nhà xuất bản Văn hóa Thông tin.

[4] Chu Nguyễn Mộng Ngọc, Hoàng Trọng (2008), "Phân tích dữ liệu nghiên cứu với SPSS”, Nhà xuất bản Thống kê.

[5] Nunnally, J. (1978), "Psycometric Theory”, New York, McGraw-Hill. 


\section{NÂNG CAO CHẤT LƯỢNG ĐÀO TẠO NGUỒN NHÂN LỰC CỦA TRƯỜNG ĐẠI HỌC CÔNG NGHIỆP}

THÀNH PHỐ HỒ CHÍ MINH TRONG BỐI CẢNH HÔ̂I NHẬP

[6] Oliver, R (1997) "Satisfaction: A behavioral perspective on the consumer", McGraw-Hill, New York.

[7] Parasuraman, Zeithaml and Berry (1985), A conceptual model of service quality and its implications for future research, Journal of Marketing, Vol. 49: 41-50.

[8] Parasuraman, Zeithaml and Berry (1991), Refinement and reassessment of SERVQUAL scale. Journal of Retailing, Vol.67: 420 - 50.

[9] Peterson, R. (1994), “A Meta-Analysis of Cronbach's Coefficient Alpha”, Journal of Consumer Research, No. 21 Vo.2.

[10] Nguyễn Đình Thọ (2011), "Phương pháp nghiên cứu khoa học trong kinh doanh”, Nhà xuất bản Lao động Xã hội.

[11] Lê Sỹ Trung (2015), "Một số giải pháp nâng cao chất lượng đào tạo của Trường Đại học Nông lâm Thái Nguyên", Tham luận tại ĐH Đại biểu Đảng bộ Trường ĐH Nông Lâm lần thứ XIV.

[12] Slater, S. (1995), "Issues in Conducting Marketing Strategy Research", Journal of Strategic.

[13] Zeithaml, Parasuraman and Berry (1990), "Delivering Quality Service”, Free Press, New York.

[14] Zeithaml and Bitner (2000), "Services marketing: Integrating customer focus across the firm", McGraw-Hill, Boston.

Ngày nhận bài: $31 / 01 / 2018$

Ngày chấp nhận đăng: 15/04/2019 